\title{
Searching for Beyond the Standard Model Physics with COHERENT Energy and Timing Data
}

\author{
Bhaskar Dutta, ${ }^{1}$ Shu Liao, ${ }^{1}$ Samiran Sinha, ${ }^{2}$ and Louis E. Strigari ${ }^{1}$ \\ ${ }^{1}$ Mitchell Institute for Fundamental Physics and Astronomy, Department of Physics and Astronomy, \\ Texas A\&M University, College Station, Texas 77843, USA \\ ${ }^{2}$ Department of Statistics, Texas A\&M University, College Station, Texas 77843, USA
}

(Received 22 April 2019; revised manuscript received 23 June 2019; published 7 August 2019)

\begin{abstract}
We search for beyond the standard model physics by combining COHERENT Collaboration energy and timing data. Focusing on light, $\lesssim \mathrm{GeV}$ mediators, we find the data favor a $\sim 10-1000 \mathrm{MeV}$ mediator, as compared to the standard model best fit at the $\lesssim 2 \sigma$ level. The best-fit coupling range is $g \sim 10^{-5}-10^{-3}$. The timing data provide statistical information on the neutrino flavor distributions that is not attainable from the nuclear recoil energies alone. This result accounts for uncertainty in the effective size of the neutron distribution, and highlights the power of including uncertainties on experimental backgrounds, nuclear structure, and beyond the standard model physics.
\end{abstract}

DOI: 10.1103/PhysRevLett.123.061801

Introduction.-The COHERENT Collaboration has reported the first detection of coherent neutrino-nucleus elastic scattering (CE $\nu$ NS ) [1]. The COHERENT experiment utilizes the Spallation Neutrino Source with a stopped-pion beam, which produces a well-known neutrino spectrum from pion and muon decay at rest. Muon neutrinos $\nu_{\mu}$ arrive from prompt charged pion decay, while $\bar{\nu}_{\mu}$ and $\nu_{e}$ are produced from the delayed muon decay. With an exposure of 14.6$308 \mathrm{~kg}$ days, the COHERENT Collaboration identified nuclear recoil events from $\mathrm{CE} \nu \mathrm{NS}$ that are well in excess of the expected background events for this exposure.

The COHERENT Collaboration data provide an important new channel to search for beyond the standard model (BSM) physics. For example, the data constrain nonstandard neutrino interactions (NSI) [2,3] due to heavy or light mediators [4-11], generalized scalar and vector neutrino interactions [12], and hidden sector models [13]. It also sets independent constraints on the effective neutron size distribution of CsI [14-16], and on sterile neutrinos [17,18]. Low mass mediator models are particularly interesting, since there are no Large Hadron Collider constraints for mediator masses $\lesssim \mathrm{GeV}$, and these models may be associated with sub$\mathrm{GeV}$ dark matter [19]. The aforementioned constraints on BSM physics have mostly been extracted from the energy distribution of nuclear recoil events.

The COHERENT Collaboration data do not directly identify the flavor components of the neutrino flux, though

Published by the American Physical Society under the terms of the Creative Commons Attribution 4.0 International license. Further distribution of this work must maintain attribution to the author(s) and the published article's title, journal citation, and DOI. Funded by SCOAP ${ }^{3}$. it is possible to make an estimate for the contribution of the different flavors. For example the prompt $\nu_{\mu}$ component may be estimated from a timing cut, while the delayed components may be extracted from their spectral signatures. Previous authors have classified the events as prompt or delayed using a two-bin analysis in timing space [20].

In this Letter, we perform a likelihood analysis on the COHERENT Collaboration data that utilizes the full energy and timing distributions of nuclear recoil events. These distributions provide information on the neutrino flavor components that is not available from the energy data alone, or when splitting the timing data into prompt and delayed events. We test for deviations from pure standard model (SM) interactions, considering as an example light mediators that couple to the SM. We show that including the timing distribution data adds substantial statistical constraining power and favors a BSM interpretation at the $\lesssim 2 \sigma$ level.

Light mediators and nonstandard interactions. - In the $\mathrm{SM}$, the differential cross section for a neutrino scattering off of a target electron or quark of mass $m$ through a $Z$ exchange is

$$
\begin{aligned}
\frac{d \sigma}{d E}= & \frac{G_{F}^{2} m}{2 \pi}\left[\left(g_{v}+g_{a}\right)^{2}+\left(g_{v}-g_{a}\right)^{2}\left(1-\frac{E}{E_{\nu}}\right)^{2}\right. \\
& \left.+\left(g_{a}^{2}-g_{v}^{2}\right) \frac{m E}{E_{\nu}^{2}}\right]
\end{aligned}
$$

where $G_{F}$ is the Fermi constant, $E$ is the recoil energy, $E_{\nu}$ is the incident neutrino energy, $\left(g_{v}, g_{a}\right)=\left(T_{3}-\right.$ $\left.2 Q_{\mathrm{em}} \sin ^{2} \theta_{W}, T_{3}\right)$ are the vector and axial-vector couplings to the $Z$ boson, $T_{3}$ is the third component of the weak isospin, $Q_{\mathrm{em}}$ is the electromagnetic charge, and $\theta_{W}$ is the 

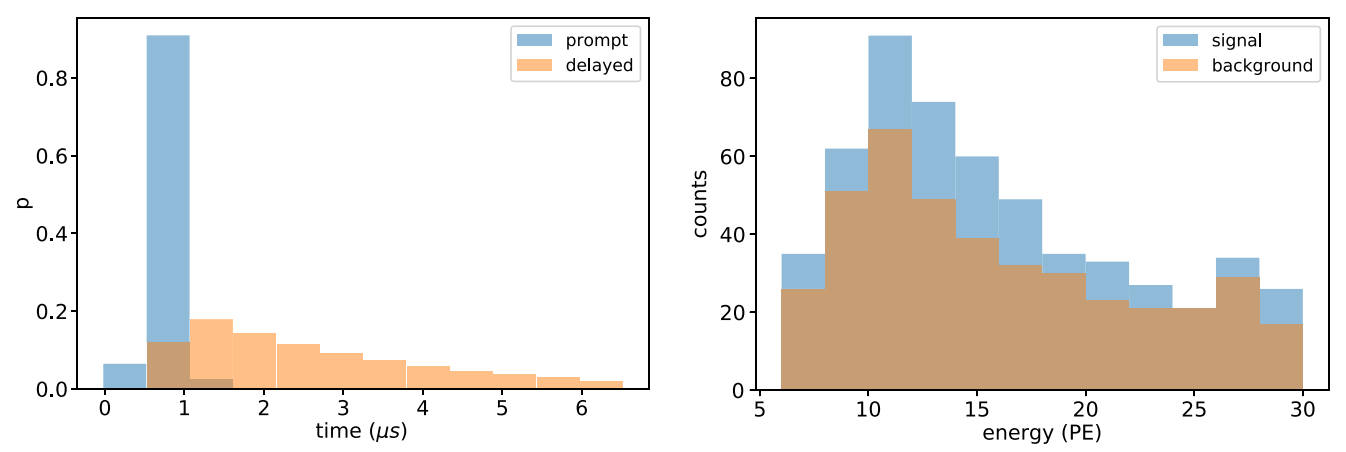

FIG. 1. Timing distribution for the prompt and delayed components (left) and energy distribution (right) as measured by the COHERENT Collaboration. The vertical axis in the time distribution gives the probability that an event is in a given time bin, while in the energy distribution it is the number of counts in that bin. In the energy distribution, the signal and background events are shown.

weak mixing angle $\left(T_{3}=-1 / 2\right.$ in our convention). For quarks in the nucleus, if the momentum transfer between the neutrino and the nucleus is smaller than or comparable to the inverse size of the nucleus [typically $E \lesssim O(50) \mathrm{MeV}$ ], coherent scattering occurs. For coherent elastic scattering off of nuclei, the axial vector contribution is proportional to the nucleus spin [21], therefore it is negligible as compared to vector contribution. The cross section for scattering on the nucleus is

$\frac{d \sigma}{d E}=\frac{G_{F}^{2} Q_{V}^{2}}{2 \pi} m_{N}\left[1-\left(\frac{m_{N} E}{E_{\nu}^{2}}\right)+\left(1-\frac{E}{E_{\nu}}\right)^{2}\right] F\left(q^{2}\right)$,

where $F\left(q^{2}\right)$ is the nuclear form factor, $Q_{V} \equiv Z\left(2 g_{v}^{u}+g_{v}^{d}\right)+$ $N\left(g_{v}^{u}+2 g_{v}^{d}\right)$, and $m_{N}$ is the nucleon mass.

The form factor is a source of uncertainty, in particular at the highest nuclear recoil energy. Since there are no direct experimental measurements of the neutron distribution in the nucleus for CsI, $F\left(q^{2}\right)$ may be estimated from the $\mathrm{CE} \nu \mathrm{NS}$ data itself. Standard parametrizations are the Helm model [22], or the symmetrized Fermi model [23]. Each of these model parametrizations are functions of the size of the neutron distribution, $R_{n}$, which can be viewed as a parameter in each model. For each of these parametrizations and including only SM contributions to the scattering rate, the best-fitting neutron radius from the COHERENT Collaboration data is $R_{n} \simeq 5.5 \mathrm{fm} \mathrm{[14].}$

The SM cross section above may be modified due to a new mediating particle, which couples to neutrinos and either electrons or quarks. For example, consider a new mediator $Z_{\mu}^{\prime}$ with the following interaction terms:

$$
\mathcal{L} \supset Z_{\mu}^{\prime}\left(g_{\nu}^{\prime} \bar{\nu}_{L} \gamma^{\mu} \nu_{L}+g_{f, v}^{\prime} \bar{f} \gamma^{\mu} f+g_{f, a}^{\prime} \bar{f} \gamma^{\mu} \gamma^{5} f\right),
$$

where $g_{\nu}^{\prime}, g_{f, v}^{\prime}$, and $g_{f, a}^{\prime}$ are constants associated with new physics. With this interaction, we can redefine the couplings $\left(g_{v}, g_{a}\right)$ of Eq. (1) in the following way,

$$
\left(g_{v}, g_{a}\right) \Rightarrow\left(g_{v}, g_{a}\right)+\frac{g_{\nu}^{\prime}\left(g_{f, v}^{\prime}, \pm g_{f, a}^{\prime}\right)}{2 \sqrt{2} G_{F}\left(q^{2}+M_{Z^{\prime}}^{2}\right)},
$$

where $q^{2}$ is the momentum transfer, $M_{Z}^{\prime}$ is the mass of the mediator, and the minus sign applies for the case of antineutrino scattering.

As an extension, we can consider the case where the quarks couple to $Z^{\prime}$ via a loop containing hidden sector particles [13]. In this case, a form factor parametrizes the quark coupling to the $Z^{\prime}$. This form factor scales as $q^{2} / \Lambda^{2}$, where $\Lambda$ is the scale in the hidden sector associated with the mass of the mediator particle that generates the quarkhidden sector interactions. In this scenario, $g_{\nu}^{\prime} g_{f}^{\prime}$ in Eq. (4) is modified to $g_{\nu}^{\prime} g_{f}^{\prime} q^{2} / \Lambda^{2}$, where $\Lambda$ is the mass scale of the hidden sector.

Motivated by the flavor content of the COHERENT Collaboration stopped pion beam, we focus on $g_{e}$ and $g_{\mu}$, associated, respectively, with electron and muon-type neutrino couplings with the $Z^{\prime}$. The up and down quark couplings to $Z^{\prime}$ are represented by $g_{u}$ and $g_{d}$, respectively, and we will assume that $g_{u}=g_{d}=g_{\nu}=g$. Under this assumption, the NSI parameter defined as $\epsilon=g_{\nu}^{\prime} g_{f, v}^{\prime} /$ $\left\{2 \sqrt{2} G_{F}\left(q^{2}+M_{Z^{\prime}}^{2}\right)\right\}=g^{2} /\left\{2 \sqrt{2} G_{F}\left(q^{2}+M_{Z^{\prime}}^{2}\right)\right\}$ is positive, or $\epsilon=g^{2} q^{2} /\left\{2 \sqrt{2} G_{F}\left(q^{2}+M_{Z^{\prime}}^{2}\right) \Lambda^{2}\right\}$ in the hidden sector scenario.

Data Analysis.-Figure 1 shows the energy and timing distribution of the COHERENT Collaboration nuclear recoil events [24]. The energy distribution is given in units of photoelectrons (PEs). Our goal is to perform a joint analysis using both the timing and energy distribution to identify possible contributions from BSM physics. To represent BSM physics, we take as parameters the couplings $g_{e}, g_{\mu}$, and mediator mass $M_{Z^{\prime}}$. To represent the uncertainty in the nuclear structure, we take $R_{n}$ as a parameter and use the Helm form factor model.

We define $\mathcal{L}$ as the likelihood function of the model parameters given the data. We assume that the sum of the observed nuclear recoil plus background counts, $N_{\text {obs }}(t, E)$, at time $t$ and energy $E$, follow a Poisson model with parameter

$$
\lambda(t, E)=(1+\alpha) N(t, E, \epsilon)+N_{\mathrm{bg}}(t, E),
$$


where $N(t, E, \epsilon)$ is the number of neutrino-induced nuclear recoil events predicted from the theory, and $N_{\mathrm{bg}}(t, E)$ denotes the true background count. Note that by definition, $N_{\mathrm{bg}}(t, E)$ is not observed. Rather, we have observed background counts, denoted by $N_{\text {obs,bg }}(t, E)$, that are proxy for the true background counts. We assume that given $N_{\text {bg }}(t, E), \quad N_{\text {obs,bg }}(t, E)$ follows a Poisson model with parameter $N_{\mathrm{bg}}(t, E)$. In the absence of prior information on $N_{\mathrm{bg}}(t, E)$, we use a noninformative prior on it, so that $\pi\left[N_{\mathrm{bg}}(t, E)\right] \propto 1$ for $N_{\mathrm{bg}}(t, E) \in[0, \infty)$.

In addition to the counts from the signal and the background components, Eq. (5) involves the uncertainty parameter $\alpha$ to account for the systematic uncertainties from flux, form factor, quenching factor, and signal acceptance uncertainties. Motivated by the results reported from the COHERENT Collaboration [1], we assume this parameter follows a normal distribution with zero mean and standard deviation $\sigma_{\alpha}=0.28$. Defining $\vec{\theta}=\left(g_{e}, g_{\mu}, m, R_{n}\right)$ as the model parameters, the most general likelihood function is

$$
\begin{aligned}
\mathcal{L}(\vec{\theta} \mid t, E) \propto & \prod_{(t, E)} \iint \exp [-\lambda(t, E)] \\
& \times \frac{\{\lambda(t, E)\}^{N_{\mathrm{obs}}(t, E)}}{N_{\mathrm{obs}}(t, E) !} \frac{\exp \left(-\alpha^{2} / 2 \sigma_{\alpha}^{2}\right)}{\sqrt{\sigma_{\alpha}^{2}}} \\
& \times \exp \left[-N_{\mathrm{bg}}(t, E)\right] \frac{\left\{N_{\mathrm{bg}}(t, E)\right\}^{N_{\mathrm{obs}, \mathrm{gg}}(t, E)}}{N_{\mathrm{obs}, \mathrm{bg}}(t, E) !} \\
& \times d \alpha d N_{\mathrm{bg}}(t, E) .
\end{aligned}
$$

In comparison to previous analyses, this likelihood explicitly includes information from the timing distribution. We take the bin width in energy space as the bin width in the space of the number of photoelectrons $n_{e}$ then convert this to recoil energy space using the relation $n_{e}=1.17(E / \mathrm{keV})$. For the timing data, we take the bin width directly from the COHERENT Collaboration data, $\sim 0.5 \mu \mathrm{s}$. In the Bayesian paradigm, we obtain the posterior probability densities for the model parameters using the MULTINEST package [25] with flat prior distributions on the parameters.

To test the robustness of our modeling in light of the steady-state (SS) background observed by the COHERENT Collaboration, we consider three separate scenarios. In the first, which we refer to as background model (a), the SS background is fixed $\left[N_{\mathrm{bg}}(t, E)=N_{\mathrm{bg}, \mathrm{obs}}\right]$, and the model parameters are only $\left(g_{e}, g_{\mu}, M_{Z^{\prime}}, R_{n}\right)$, and we do not need to integrate over the background in Eq. (6). In the second, which we refer to as background model (b), we take the background shape from the COHERENT Collaboration data, but allow for an overall scaling of the background to account for an uncertainty in the normalization. For model (b), we assume that the total number of background counts follow a Poisson distribution. Instead of integrating over the background for each bin, we integrate over the total number of background counts in this scenario. In the third, which we refer to as background model (c), we take the background to be a Poisson model in each bin, and integrate over the predicted number of events in each bin according to Eq. (6). For models (a) and (b), in order to mitigate a bias in our reconstructed parameters, we exclude bins in which there are zero background counts. Using simulated data with the same number of signal and background counts as in the COHERENT data, we have verified that excluding the bins with zero background counts does not bias the reconstruction of the couplings and the mediator mass.

For comparing two models, say models 0 and 1 , where one is nested within another, we use the likelihood ratio test within a frequentist paradigm. We consider the test statistic $U=-2\left[\log \left(\mathcal{L}_{0}\right)-\log \left\{\mathcal{L}_{1}(\hat{\theta})\right\}\right]$, where we define $\log \left(\mathcal{L}_{0}\right)$ as the log-likelihood for model 0 , in which only $R_{n}$ is free and the other three parameters are set to zero, and $\log \left\{\mathcal{L}_{1}(\hat{\theta})\right\}$ is the log-likelihood for the model, in which at least one of the parameters in $\left(g_{e}, g_{\mu}, M_{Z^{\prime}}\right)$ is free. For the latter model, $\hat{\theta}$ denotes the maximum likelihood estimator of $\vec{\theta}$, and $\hat{\theta}$ is obtained by maximizing the marginal likelihood, Eq. (6), where $N_{\mathrm{bg}}(t, E)$ is integrated out. The $p$ value of the test is $p=\operatorname{pr}\left(\chi_{\eta}^{2}>U_{\mathrm{obs}}\right)$, where $U_{\mathrm{obs}}$ is the observed value of $U$ and $\chi_{\eta}^{2}$ is the chi-square distribution with $\eta$ degree of freedom. Here, $\eta$ is the difference between the number of estimated parameters in models 0 and 1. A small $p$ value provides significant evidence against the null hypothesis (SM). Applying this general procedure, we can test if a model parameter (i.e., $g_{\mu}$ or $g_{e}$ ) is positive. The corresponding significance $Z$ is $\Phi^{-1}(1-p / 2)$, where $\Phi^{-1}$ is the inverse cumulative distribution function of the standard normal distribution. Significant results can also be seen through a large value of $Z$.

Results.-We begin by considering how well $R_{n}$ is determined for each of our three different background models. The posterior probability distributions for $R_{n}$ are shown in the left panel of Fig. 2 for each of these background models. For background models (a) and (b), the best-fit $R_{n}$ is slightly lower than that from model (c), though it is still statistically consistent with the result from model (c).

In order to simplify our analysis, for our BSM scenario we consider models with couplings such that $g_{\nu}=g_{u}=$ $g_{d}=g^{\prime}$. To compare the sensitivity of the data to different flavors, we either fit for $g_{\mu}$ and fix $g_{e}$, or vice versa. The key features of our analysis are unchanged if we use a different relation among the couplings.

In Fig. 3, we show the resulting posterior probability distributions for $g_{\mu}$ (assuming that $g_{e}=0$ ), for the cases of $M_{Z^{\prime}}=10$ and $1000 \mathrm{MeV}$. We show the result for background model (c), in which the background is taken to be a Poisson model in each energy and time bin. The figures contain distributions using energy information alone, and distributions using both energy and timing 

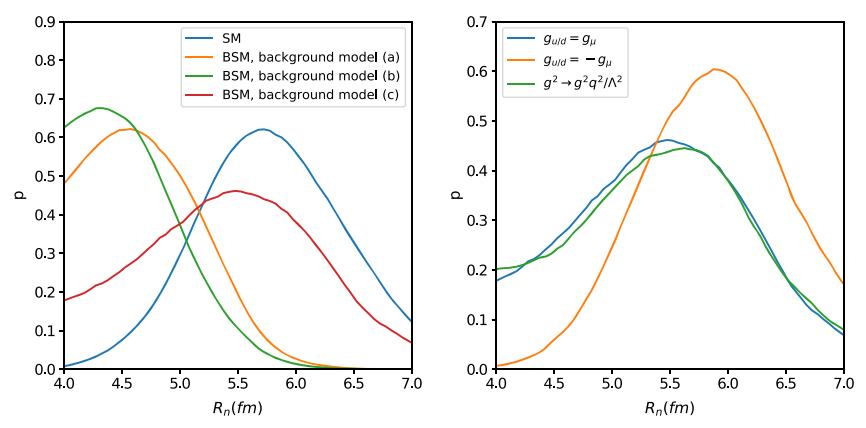

FIG. 2. Posterior probability distributions for the effective size of the nucleus $R_{n}$ for different background models (left), and for BSM models (right). The (blue) SM distribution takes $R_{n}$ as a parameter, and the BSM distribution takes $\left(R_{n}, g^{\prime}\right.$, and $\left.M_{Z^{\prime}}\right)$ as free parameters.

information. For both $M_{Z^{\prime}}=10$ and $1000 \mathrm{MeV}$, the $g_{\mu}$ distributions are better constrained when including timing data, and deviate from the SM prediction that these couplings are zero.

We find that our results are relatively insensitive to the assumed background model. To quantify this, in Table I, we show the significance $Z$ for each of the three different background assumptions. In Table I, we also show the corresponding values of $Z$ for an analysis with the energy data alone. Here, we find that statistical significance of the
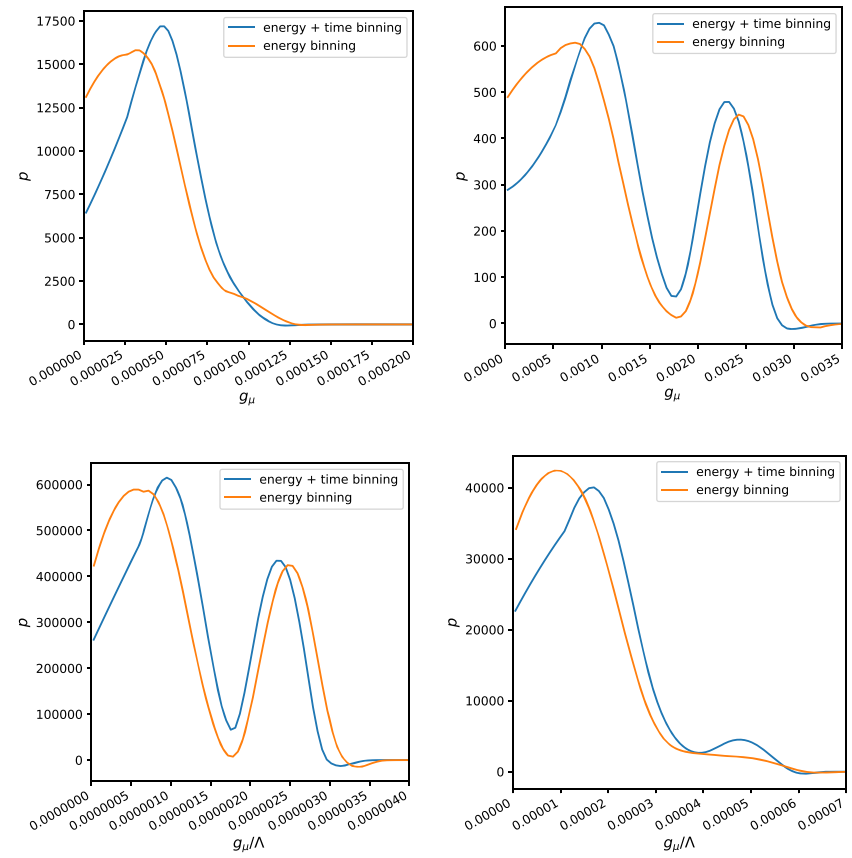

FIG. 3. Posterior probability distributions for $g_{\mu}$ (top row) or $g_{\mu} / \Lambda$ (bottom row, if there is form factor), using the energy data alone (orange) and using the combined energy and timing data (blue). The left column assumes a mediator mass of $M_{Z^{\prime}}=10 \mathrm{MeV}$, and the right column assumes a mediator mass of $M_{Z^{\prime}}=1000 \mathrm{MeV}$.
TABLE I. Significance in the likelihood ratio test for different background models defined in the text. The first column gives mediator mass, with the first row taking the mediator mass as a free parameter. All entries assume $g_{e}=0$ and $g_{\mu} \neq 0$. Each primary entry is obtained using the combined energy and timing data, and the numbers in parenthesis use just the energy data.

\begin{tabular}{lccc}
\hline \hline $\begin{array}{l}\text { Mediator mass, } M_{Z^{\prime}} \\
(\mathrm{MeV})\end{array}$ & $\begin{array}{c}\text { Fixed } \\
\text { [model (a)] }\end{array}$ & $\begin{array}{c}\text { Fixed shape } \\
\text { [model (b)] }\end{array}$ & $\begin{array}{c}\text { Varying } \\
\text { [model (c)] }\end{array}$ \\
\hline Free & $1.4(0.7)$ & $0.9(0.6)$ & $1.1(0.6)$ \\
10 & $1.9(1.2)$ & $1.4(1.1)$ & $1.6(1.0)$ \\
100 & $1.9(1.1)$ & $1.4(1.1)$ & $1.6(1.1)$ \\
1000 & $1.9(1.2)$ & $1.4(1.1)$ & $1.6(1.1)$ \\
\hline \hline
\end{tabular}

deviation from the SM is lower for all background models, as compared to the analysis that utilizes both energy and timing data. The results in Table I show that the timing data provides additional information on the flavor content of the fluxes that is not provided by the energy data alone. The bin-by-bin likelihood analysis that we employ is able to statistically separate the prompt and delayed distributions, with the timing information more strongly constraining the prompt $g_{\mu}$ component. In contrast, the $g_{e}$ component only contributes to delayed neutrino recoil spectrum, and is less well constrained when adding in the timing information.

Figure 4 shows the probability density in $\log _{10}\left(M_{Z^{\prime}}\right)$ vs $\log _{10}\left(g_{\mu}\right)$ or $\log _{10}\left(g_{\mu} / \Lambda\right)$ space. The shape of the boundary in both plots can be understood as follows: in the small mediator mass region, $q^{2} \gg M_{Z^{\prime}}^{2}$, the NSI parameter is independent of the small mediator mass, while in the large mediator mass region, $M_{Z^{\prime}}^{2} \gg q^{2}$, the NSI parameter depends on $g^{2} / M_{Z^{\prime}}^{2}$, thus, in $\log$ space, the slope is about 1 .

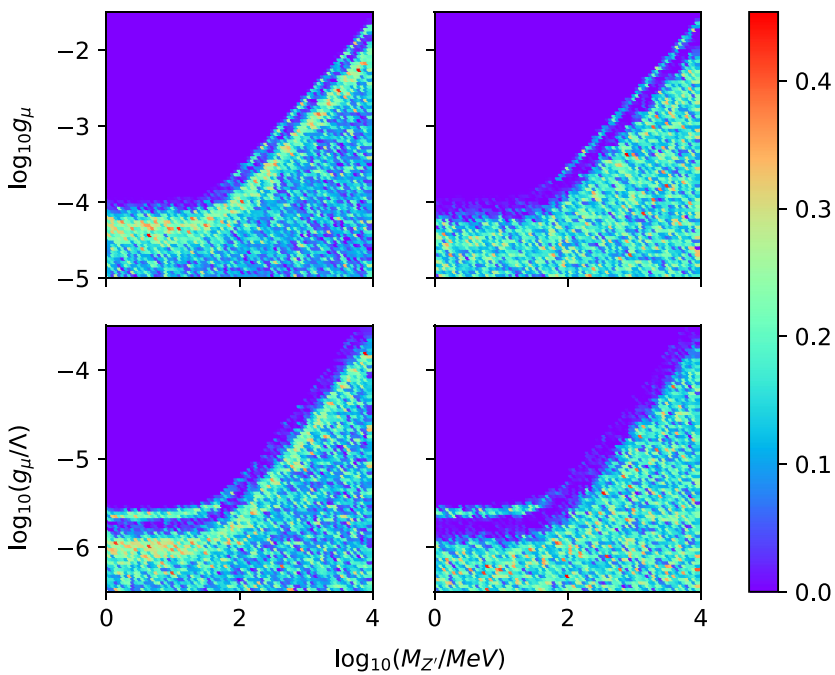

FIG. 4. Heat map of the probability density in $\log _{10}\left(M_{Z^{\prime}}\right)$ vs $\log _{10} g_{\mu}$ (top row) and $\log _{10}\left(M_{Z^{\prime}}\right)$ vs $\log _{10}\left(g_{\mu} / \Lambda\right.$ ) (bottom row, for a form factor $q^{2} / \Lambda^{2}$ ) parameter space using energy and timing data (left) and energy data alone (right). 
TABLE II. The $1 \sigma$ constraining range on $g_{\mu}$ and $g_{e}$ using energy plus timing information.

\begin{tabular}{lccc}
\hline \hline$M_{Z^{\prime}}(\mathrm{MeV})$ & 10 & 100 & 1000 \\
\hline$g_{\mu}$ & {$[1.87,6.65] \times 10^{-5}$} & {$[0.41,1.47] \times 10^{-4} \oplus[2.47,2.66] \times 10^{-4}$} & {$[0.48,1.32] \times 10^{-3} \oplus[2.17,2.47] \times 10^{-3}$} \\
$g_{e}$ & {$[0,6.12] \times 10^{-5}$} & {$[0,1.53] \times 10^{-4} \oplus[2.53,2.84] \times 10^{-4}$} & {$[0,1.22] \times 10^{-3} \oplus[2.22,2.77] \times 10^{-3}$} \\
\hline \hline
\end{tabular}

The isolated island at large mediator mass region is because the global degeneracy for the weak charge across all energy bins (since the NSI parameter is independent of energy); thus, the constraint on the charge $Q_{v}$ in Eq. (2) results in two solutions for $g$. On the contrary, if a hidden sector is introduced to generate a form factor $\sim q^{2} / \Lambda^{2}$, the NSI parameter becomes independent of energy in the smaller mass region and consequently, the degeneracy appears in the smaller mass region.

The above results favoring $g \neq 0$ are independent of the uncertainty in the assumed value for $R_{n}$, since we have taken $R_{n}$ as a parameter in our analysis. It is, however, interesting to determine the manner in which $R_{n}$ is degenerate with the BSM model. As an example, we can compare to the case in which $g_{u}=g_{d}=-g_{\nu}=g$, as in this case, there is destructive interference with the SM weak charge. The resulting $R_{N}$ distribution with this assumption is shown in the right-hand panel of Fig. 2. Here, we see that the degeneracy is such that larger $R_{N}$ is now more favored, though the results are statistically consistent with the assumption of $g_{u}=g_{d}=g_{\nu}=g$.

To quantify the constraints on the NSI parameters in Fig. 3, we show in Table II the $1 \sigma$ window of $g_{\mu}$ and $g_{e}$ assuming 10, 100, and $1000 \mathrm{MeV}$ mediator masses using our results from the energy and timing analysis. Note that this range is consistent with general constraints on the couplings and masses of light mediators [26].

Constraints on the NSI parameters also come from neutrino oscillation experiments. To obtain constraints from neutrino oscillation experiments, the NSI parameters are expressed as $\epsilon=g^{2} /\left\{2 \sqrt{2} G_{F}\left(M_{Z^{\prime}}^{2}\right)\right\}$. The existing constraints from the neutrino oscillation experiments are consistent with our result, within the $\sim 2 \sigma$ range [4]. For example, the allowed range for a heavy mediator for $\epsilon_{\mu \mu}^{u}$ is $[0,0.005]$ (our model only has positive $\epsilon$ ). This converts to $g=[0,0.0013]$ at $M_{Z^{\prime}}=1 \mathrm{GeV}$, which covers the $1 \sigma$ region as shown in Fig. 3.

There also exists a so-called LMA-dark solution, which is characterized by larger NSI couplings and is not strongly constrained by oscillation experiments. Reference [20] discusses this scenario specifically in the context of the COHERENT Collaboration data. Since the SM is not nested within the LMA-Dark solution, to establish significance in this case, we must appeal to the Bayes factor. We find that the Bayes factor is 1.12(0.85) with (without) timing information, which means this kind of NSI is also slightly preferred over SM if we include the timing data.

Discussion and Conclusion.-We have performed a fit to the energy and timing distribution of nuclear recoil events from the COHERENT Collaboration data. We have shown that including the information in both the energy and timing distributions of the COHERENT Collaboration data, there is a $\sim 2 \sigma$ deviation between the best-fitting model and the SM prediction. Light mediators in the mass range $\sim 10-1000 \mathrm{MeV}$ are able to provide a good fit to the data. In the appropriate limits of large mediator mass, $\gtrsim 1 \mathrm{GeV}$, it is equivalent to a heavy mediator NSI analysis that has been performed by the COHERENT Collaboration and by previous authors. This result is consistent with other experiments searching for dark photons, as well as oscillation experiments.

Our analysis motivates an event-by-event analysis of COHERENT Collaboration nuclear recoil events. Given information on individual nuclear recoil events, our analysis may be extended in the future by considering an unbinned limit to the likelihood, and may include appropriate uncertainty on the energy and time of an event. Future data from the COHERENT Collaboration [27] will better clarify the results that we have obtained.

B. D. and L. E. S. acknowledge support from U.S. DOE Grant No. DE-SC0010813. We acknowledge support from COS-STRP (TAMU), and would like to thank Grayson Rich and Kate Scholberg for discussions on this Letter.

[1] D. Akimov et al. (COHERENT Collaboration), Science 357, 1123 (2017).

[2] T. Ohlsson, Rep. Prog. Phys. 76, 044201 (2013).

[3] O. G. Miranda and H. Nunokawa, New J. Phys. 17, 095002 (2015).

[4] P. Coloma, P. B. Denton, M. C. Gonzalez-Garcia, M. Maltoni, and T. Schwetz, J. High Energy Phys. 04 (2017) 116.

[5] P. Coloma, M. C. Gonzalez-Garcia, M. Maltoni, and T. Schwetz, Phys. Rev. D 96, 115007 (2017).

[6] J. Liao and D. Marfatia, Phys. Lett. B 775, 54 (2017).

[7] J. B. Dent, B. Dutta, S. Liao, J. L. Newstead, L. E. Strigari, and J. W. Walker, Phys. Rev. D 97, 035009 (2018).

[8] J. Billard, J. Johnston, and B. J. Kavanagh, J. Cosmol. Astropart. Phys. 11 (2018) 016.

[9] M. Lindner, W. Rodejohann, and X.-J. Xu, J. High Energy Phys. 03 (2017) 097.

[10] Y. Farzan, M. Lindner, W. Rodejohann, and X.-J. Xu, J. High Energy Phys. 05 (2018) 066.

[11] V. Brdar, W. Rodejohann, and X.-J. Xu, J. High Energy Phys. 12 (2018) 024.

[12] D. Aristizabal Sierra, V. De Romeri, and N. Rojas, Phys. Rev. D 98, 075018 (2018). 
[13] A. Datta, B. Dutta, S. Liao, D. Marfatia, and L. E. Strigari, J. High Energy Phys. 01 (2019) 091.

[14] E. Ciuffoli, J. Evslin, Q. Fu, and J. Tang, Phys. Rev. D 97, 113003 (2018).

[15] D. Aristizabal Sierra, J. Liao, and D. Marfatia, J. High Energy Phys. 06 (2019) 141.

[16] D. K. Papoulias, T. S. Kosmas, R. Sahu, V. K. B. Kota, and M. Hota, arXiv:1903.03722.

[17] T. S. Kosmas, D. K. Papoulias, M. Tortola, and J. W. F. Valle, Phys. Rev. D 96, 063013 (2017).

[18] C. Blanco, D. Hooper, and P. Machado, arXiv:1901.08094.

[19] R. Essig et al., in Proceedings, 2013 Community Summer Study on the Future of U.S. Particle Physics: Snowmass on the Mississippi (CSS2013): Minneapolis, MN, USA, 2013 (2013).
[20] P. B. Denton, Y. Farzan, and I. M. Shoemaker, J. High Energy Phys. 07 (2018) 037.

[21] J. Barranco, O. G. Miranda, and T. I. Rashba, J. High Energy Phys. 12 (2005) 021.

[22] J. D. Lewin and P. F. Smith, Astropart. Phys. 6, 87 (1996).

[23] J. Piekarewicz, A. R. Linero, P. Giuliani, and E. Chicken, Phys. Rev. C 94, 034316 (2016).

[24] D. Akimov et al. (COHERENT Collaboration), arXiv:1804 .09459 .

[25] F. Feroz, M. P. Hobson, and M. Bridges, Mon. Not. R. Astron. Soc. 398, 1601 (2009).

[26] M. Bauer, P. Foldenauer, and J. Jaeckel, J. High Energy Phys. 07 (2018) 094.

[27] D. Akimov et al. (COHERENT Collaboration), arXiv: 1803.09183. 